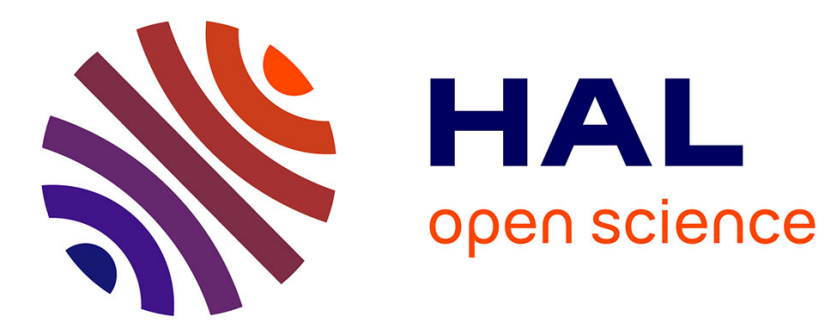

\title{
Placental infection by Zika virus in French Guiana
}

Léo Pomar, Véronique Lambert, Yoann Madec, Manon Vouga, Céline Pomar, Severine Matheus, Arnaud Fontanet, Alice Panchaud, Gabriel Carles, David Baud

\section{- To cite this version:}

Léo Pomar, Véronique Lambert, Yoann Madec, Manon Vouga, Céline Pomar, et al.. Placental infection by Zika virus in French Guiana. Ultrasound in Obstetrics and Gynecology = Ultrasound in Obstetrics \& Gynecology, 2019, 10.1002/uog.21936 . pasteur-02863590

\section{HAL Id: pasteur-02863590}

https://hal-pasteur.archives-ouvertes.fr/pasteur-02863590

Submitted on 15 Jun 2020

HAL is a multi-disciplinary open access archive for the deposit and dissemination of scientific research documents, whether they are published or not. The documents may come from teaching and research institutions in France or abroad, or from public or private research centers.
L'archive ouverte pluridisciplinaire HAL, est destinée au dépôt et à la diffusion de documents scientifiques de niveau recherche, publiés ou non, émanant des établissements d'enseignement et de recherche français ou étrangers, des laboratoires publics ou privés.

\section{(ㅇ)(1) $\$$}

Distributed under a Creative Commons Attribution - NonCommercial - NoDerivatives 44.0 


\section{Placental infection by Zika virus in French Guiana}

Léo Pomar ${ }^{1,2}$, M.Sc., Véronique Lambert ${ }^{2}$, M.D., Yoann Madec ${ }^{3}$, Ph.D., Manon Vouga ${ }^{1}$, M.D., Ph.D., Céline Pomar², R.M., Séverine Matheus ${ }^{4}$, Ph.D., Arnaud Fontanet ${ }^{3,5}$, M.D., Ph.D., Alice Panchaud $^{6}$, Ph.D., Gabriel Carles², M.D., David Baud ${ }^{1}$, M.D., Ph.D.

${ }^{1}$ Materno-fetal and Obstetrics Research Unit, Department “Femme-Mère-Enfant”, University Hospital, Lausanne, Switzerland

${ }^{2}$ Department of Obstetrics and Gynecology, Centre Hospitalier de l'Ouest Guyanais Franck Joly, Saint-Laurent-du-Maroni, France

${ }^{3}$ Emerging Diseases Epidemiology Unit, Institut Pasteur, Paris, France

${ }^{4}$ Laboratory of Virology, National Reference Center for Arboviruses, Institut Pasteur, Cayenne; Environment and infections risks unit, Institut Pasteur, Paris, France

${ }^{5}$ PACRI Unit, Conservatoire National des Arts et Métiers, Paris, France

${ }^{6}$ School of Pharmaceutical Sciences, Geneva University and Service of Pharmacy, Lausanne University Hospital, Lausanne, Switzerland

*Corresponding author:
Prof David Baud, MD PhD

Materno-fetal \& Obstetrics Research Unit

Department of Obstetrics and Gynecology

Centre Hospitalier Universitaire Vaudois (CHUV)

1011 Lausanne - SWITZERLAND

Email: david.baud@chuv.ch 
Keywords: Zika, congenital infection, placentomegaly, placental pathology, ultrasound Short title: Placental infection by Zika virus 


\section{Contribution:}

What are the novel findings of this work?

Zika virus (ZIKV) vertical transmission is not systematic and does not always lead to placental dysfunction with associated adverse outcomes. This original research presents the placental findings on prenatal ultrasound and anatomopathological examinations in a large cohort of ZIKV-infected placentas and controls.

What are the clinical implications of this work?

ZIKV-infected placentas exhibit a higher risk of placentomegaly and pathological anomalies than non-infected placentas, and early placentomegaly may represent the first sign of congenital ZIKV infection, which could be particularly useful in low income countries where the access to tertiary centers may be restricted. 


\section{ABSTRACT}

Objectives: To correlate placental thickness during pregnancy and histopathological results with placental Zika virus (ZIKV) infection.

Methods: During the ZIKV epidemic in French Guiana, trans-placental contamination was defined either by a positive RT-PCR or identification of specific IgM in at least one placental, fetal or neonatal sample. Placentas were classified as non-exposed (from non-infected pregnant woman), exposed (from ZIKV-infected pregnant women without trans-placental contamination) or infected (from ZIKV-infected pregnant women with proven trans-placental contamination). Placentas were assessed by monthly prenatal ultrasound, measuring placental thickness and umbilical artery Doppler, and anatomopathologic examination after birth or IUFD. Placental thickness during pregnancy and anatomopathologic findings were correlated to the ZIKV-status of the placenta.

Results: Among 291 fetuses/placentas from proven infected mothers, trans-placental infection was confirmed in 76 cases, of which 16 resulted in Congenital Zika Syndrome (CZS) and 11 in fetal loss. The 215 remaining placentas without evidence of ZIKV infection represented the exposed group. A total of 334 placentas from ZIKV-negative pregnant women represented the non-exposed group. Placentomegaly (thickness $>40 \mathrm{~mm}$ ) was observed more frequently in infected placentas (39.5\%) compared to exposed placentas (17.2\%) or controls (7.2\%), even when considering gestational age at diagnosis and co-morbidities (adjusted Hazard Ratio [aHR] 2.02 [95\%CI 1.22-3.36] and aHR 3.23 [95\%CI 1.86-5.61], respectively), and appeared earlier in infected placentas. Placentomegaly was observed even more frequently in case of CZS (62.5\%) or fetal loss (45.5\%) compared to asymptomatic congenital infection (30.6\%) (aHR 5.43 [95\%CI 2.17-13.56] and aHR 4.95 [95\%CI 1.65-14.83], respectively). Umbilical artery Doppler anomaly was observed more frequently in case of trans-placental infection resulting in fetal loss (30.0\% vs 6.1\%; adjusted Relative Risk [aRR] 4.83 
[95\%CI 1.09-20.64]). Infected placentas also exhibited a higher risk of any pathological anomalies than exposed placentas (aRR 2.60 [1.40-4.83]).

Conclusions: Early placentomegaly may represent the first sign of trans-placental contamination and should lead to an enhanced follow-up of these pregnancies. 


\section{INTRODUCTION}

The recent worldwide epidemic has confirmed vertical trans-placental transmission of Zika virus (ZIKV) and its association with congenital anomalies, in particular severe central nervous system (CNS) lesions ${ }^{1-3}$.

In other congenital viral infections, the placenta is the major route of maternal-fetal transmission, through which the virus spreads during maternal viremia ${ }^{4,5}$. Viral replication in the placenta could impair vascular remodeling and cause fibrosis, leading to placental dysfunction and reducing maternal-fetal circulation ${ }^{6-8}$. A recent in vivo study on non-immune pregnant primates infected by ZIKV demonstrated a robust maternal-placental-fetal inflammatory response associated with abnormal oxygen transport within the placenta ${ }^{9}$. Placentitis and placentomegaly are also described in human placentas infected by ZIKV ${ }^{10-12}$. Studies reporting on features of congenital ZIKV described both CNS and non-CNS lesions, which could be a consequence of fetal and/or placental infection $^{3,13,14}$.

Similar to other TORCH group infections, vertical transmission is not systematic and does not always lead to placental dysfunction with associated adverse outcomes. In a previous study investigating the rates of maternal-fetal infection, we demonstrated transplacental infection by ZIKV in 76/291 (26.1\%) fetuses/neonates born from infected mothers, of which 27 (35.5\%) exhibited severe adverse outcomes (11 fetal losses and 16 severe CNS anomalies suggestive of Congenital Zika Syndrome $[\mathrm{CZS}])^{15}$.

Here, we describe the placental findings on prenatal ultrasound and anatomopathological examinations and correlate them with ZIKV congenital infection and severe adverse outcomes (fetal loss or CZS). 


\section{MATERIALS AND METHODS}

\section{Study population}

Details of the study protocol are described elsewhere ${ }^{14,15}$. Briefly, the study was conducted at the French Guiana Western Hospital Center (Centre Hospitalier de l'Ouest Guyanais - CHOG) at the beginning of ZIKV epidemic from January 1-July 15, 2016.

Initial inclusion of ZIKV-infected pregnant women and controls occurred either through routine serological testing of all pregnant women admitted to the prenatal diagnosis unit of the CHOG (performed in each trimester of pregnancy and at birth) or through serological and molecular testing in the presence of maternal symptoms. Molecular and serological testing were performed at the French Guiana National Reference Center (NRC) for arboviruses (Pasteur Cayenne), using the Realstar Zika Kit (Altona Diagnostics GmbH, Hamburg, Germany) for RT-PCR, in-house MACELISA and micro-neutralizing assays for serologies.

Patients were monitored per the clinical standard of care in France, with the exception of prenatal ultrasound (US) performed monthly for patients positive for ZIKV, and enhanced by two supplementary ultrasounds for patients negative for ZIKV (at 26-28 weeks’ gestation [wg] and 3638wg), as recommended by the French authorities and others ${ }^{16-18}$. Data regarding demographic, medical, and obstetrical characteristics and possible risk factors for congenital diseases were prospectively collected. The study protocol was approved by the institutional review board of the CHOG.

\section{Study groups by placental exposure status}


Pregnant women were defined as ZIKV-positive either by a positive RT-PCR result in blood and/or urine, or by the presence of specific ZIKV IgM after anti-ZIKV antibody detection in the blood, confirmed by a micro-neutralizing assay in cases of suspected co-infections with other arboviruses (expected to be minimal, as circulation of Dengue virus has been low in French Guiana since 2014). A confirmed congenital ZIKV infection was defined either by ZIKV RNA amplification by RT-PCR from at least one fetal/neonatal sample (placenta, amniotic fluid, cerebrospinal fluid, urine or blood) or identification of ZIKV specific IgM in the umbilical cord/neonatal blood or in cerebrospinal fluid. Placenta ZIKV infection status was classified into three categories as follow:

1) Control placentas stemming from pregnant women who tested negative for ZIKV up to delivery,

2) Exposed placentas stemming from proven ZIKV-infected pregnant women without reported congenital infection in the newborn (i.e. “expZIKV”),

3) Infected placentas stemming from proven ZIKV-infected pregnant women with proven congenital infection in the newborn (i.e. “congZIKV”)

Infected placentas were then classified into placentas resulting from CZS, fetal loss or asymptomatic congenital infection at birth.

\section{Placental analysis}

Sonographers, clinicians and pathologists were aware of the ZIKV-status of the pregnant women, as well as potential fetal malformations at the time of prenatal and postnatal placental examination but were unaware of the fetal virological status for ZIKV (defined after postnatal testing).

\section{Prenatal placental examination}

All prenatal US examinations were performed by two experienced sonographers (VL, LP) using E8 and E10 Voluson ultrasounds with abdominal (RM6C) and transvaginal (RIC5-9-D) transducers 
(General Electric Medical System, Milwaukee, USA). Placental maximum vertical thickness was measured at each prenatal US, by longitudinal (non-oblique) scanning, with the US probe and beam perpendicular to the chorial plate. The measurements avoided areas of amniochorial detachment and periods of uterine contractility. Placentomegaly was defined as a placental thickness greater than $40 \mathrm{~mm}^{19,20}$. Umbilical artery Doppler was performed on a free vertical loop of cord at each prenatal US for ZIKV-infected pregnant women. Resistance index (RI) was reported and correlated with gestational age. An abnormal umbilical artery Doppler was defined as a RI higher than the $95^{\text {th }}$ percentile ${ }^{21}$.

\section{Post-natal placental examination}

Routine examination of the placenta at the time of delivery was completed by a midwife and/or an obstetrician following live birth or intra-uterine fetal demise (IUFD). Anatomopathological examination of the placenta was intended to be offered to all patients with a confirmed ZIKV infection during pregnancy. During the peak of the epidemic, however, this was limited due to a lack of storage capacity, fixation material and pathologist availability. The placentas were fixed in $10 \%$ buffered formalin for 48 hours. Placental weight was measured and a birthweight / placental weight ratio was calculated using the Thompson et al. method ${ }^{22}$. Low and high birthweight / placental weight ratios were defined by a ratio lower than the third percentile, and higher than the ninety-seventh percentile, respectively (according to gestational age and sex) ${ }^{22}$. Samples from the umbilical cord, membrane, and placental parenchyma, including the decidua and chorionic plates were collected. Specific immunochemistry examination was offered for cases with congenital Zika virus syndrome (CZS) identified prenatally. 
Anatomopathological anomalies included abnormal birthweight / placental weight ratio, signs of placental inflammation (chorioamnionitis lesions, villitis and intervillitis, calcifications), infarcts, ischemic necrosis with fibrin deposits, thrombosis, leukocytic infiltration and Hofbauer cell hyperplasia (defined in Appendix 1).

\section{Covariates}

Gestational age was collected at each prenatal measurement of placental thickness and at the time of delivery (after birth or IUFD). Maternal comorbidities (diabetes, vascular pathologies, thrombophilia, severe anemia, lead-poisoning, alcohol consumption, malnutrition, vitamin K deficit) or co-infections (TORCH group infections) and risk of aneuploidy $>1 / 250$ were evaluated as effect modifiers for the association between placental thickness, Doppler abnormalities or pathological results and ZIKVstatus of the placenta.

\section{Statistical analyses}

Baseline maternal and fetal / neonatal characteristics were compared based on the ZIKV-status of the placenta. Categorical variables were compared using Chi2 or Fisher's exact test, while the distribution of continuous variables was compared using the Krustall-Wallis test.

Placental thickness measurements were compared every month across the three groups (congZIKV, expZIKV and controls) using ANOVA. The weekly incidence of placentomegaly ( $>40 \mathrm{~mm}$ thickness) was compared across the three groups using Kaplan-Meier curves, and the cumulative incidence was compared using the logrank test. The weekly incidence of placentomegaly was also compared across fetuses with CZS, fetal loss or asymptomatic congenital infection at birth, using the same statistical methods. Factors associated with the incidence of placentomegaly throughout pregnancy were 
evaluated using the Cox proportional hazards model. Factors associated with abnormal umbilical Doppler measurements or anatomopathological findings, including placental ZIKV status, were evaluated using a logistic regression model. Abnormal Doppler measurements and anatomopathological findings were also compared across fetuses with CZS, fetal loss or asymptomatic congenital infection at birth, using the same statistical methods. Confidence intervals on risk-ratios and hazard ratios were calculated using the exact method. When data are missing, denominators are presented. All statistical analyses were conducted using Stata 14 (Stata Corporation, College Station, TX, USA). 


\section{RESULTS}

From January 1 to July 15, 2016, a total of 1,690 pregnant women were tested for ZIKV during their pregnancy, among which 498 had confirmed maternal ZIKV infection (Figure 1). Among 291 fetuses/neonates/placentas with available testing for ZIKV from the proven infected mothers, congenital ZIKV infection was confirmed in 76 (26.1\%) cases and represented the infected group (congZIKV), of which 27/76 (35.5\%) resulted in fetal loss ( $\mathrm{n}=11)$ or CZS ( $\mathrm{n}=16)$. The remaining 215 fetuses/neonates/placentas without evidence of ZIKV infection (73.9\%) represented the exposed group (expZIKV). A total of 334 fetuses/neonates/placentas from mothers that tested negative for ZIKV throughout their pregnancy represented the control group.

As shown in Table 1, no differences in baseline maternal characteristics and birth parameters were observed between these three groups. Fetal loss was more prevalent in cases of congenital (congZIKV) infection (14.5\% vs $0.5 \%$ and $1.2 \%, \mathrm{p}<0.001)$. The timing of diagnosis of maternal ZIKV infection was similar between infected (congZIKV) and exposed (expZIKV) placentas.

\section{Association between prenatal ultrasound findings and ZIKV status of the placenta}

Placental thickness was measured at least once during pregnancy, with a median number of 4,4 and 3 measurements for infected (congZIKV), exposed (expZIKV) and control placentas respectively. By gestational age, infected placentas (congZIKV) tended to be thicker than exposed placentas (expZIKV) between 18 and 22 weeks $(p=0.05)$ and were significantly thicker after 26 weeks' gestation $(\mathrm{p}<0.001)$. Similarly, infected placentas (congZIKV) were significantly thicker than 
controls after 18 weeks $(\mathrm{p}<0.001)$ (Figure 2). The kinetics of placental thickness by gestational age, between controls, expZIKV and congZIKV placentas is shown in the supplementary figure 1.

Placentomegaly (>40 mm thickness, supplementary figure 2) was observed more frequently in infected placentas (39.5\%) compared to exposed placentas (17.2\%) or controls (7.2\%), even when adjusting for gestational age at diagnosis and co-morbidities (2.20\% vs $0.79 \%$ for expZIKV vs $0.43 \%$ for controls per week, aHR 2.02 [95\%CI 1.22-3.36] p=0.007 and aHR 3.23 [95\%CI 1.86-5.61] $\mathrm{p}<0.001$, when congZIKV were compared to expZIKV and controls, respectively) (Figure 3). Placentomegaly was not more frequent in exposed placentas compared to controls after adjustment for gestational age and co-morbidities $(\mathrm{aHR}=1.34$ [95\%CI 0.78-2.29] $\mathrm{p}=0.288)$. Placentomegaly appeared earlier in infected placentas (congZIKV, as early as 18 weeks, median 30 weeks) compared to exposed placentas (expZIKV, as early as 23 weeks, median 33 weeks) and to controls (as early as 22 weeks, median 34 weeks) $(\mathrm{p}<0.001)$.

When considering infected placentas, placentomegaly was observed more frequently in placentas from pregnancies with CZS (10/16, 62.5\%) or fetal loss (5/11, 45.5\%) compared to placentas from pregnancies with asymptomatic congenital ZIKV infection (15/49, 30.6\%), even when adjusting for gestational age at diagnosis and co-morbidities (4.09\% and $3.14 \%$ vs $1.48 \%$ per week, aHR 5.43 [95\%CI 2.17-13.56] $\mathrm{p}<0.0001$ and aHR 4.95 [95\%CI 1.65-14.83] $\mathrm{p}=0.004$ ) (Figure 4). Placentomegaly appeared earlier in CZS (as early as 18 weeks) and fetal losses (as early as 19 weeks) compared to asymptomatic congenital infections (26 weeks) ( $\mathrm{p}=0.044$ and $\mathrm{p}=0.048$, respectively). When considering placentomegaly $>40 \mathrm{~mm}$ as a predicter for congenital ZIKV-infection, the sensitivity was $39.5 \%$, the specificity $88.8 \%$, the positive predictive value (PPV) $32.3 \%$, and the negative predictive value (NPV) 91.3\%. As a predictor for CZS, the sensitivity was 62.5\%, the specificity $86.7 \%$, the PPV $11.0 \%$ and the PPV 98.9\%. As a predictor for fetal loss related to 
congenital ZIKV infection, the sensitivity was $45.5 \%$, the specificity $86.0 \%$, the PPV $5.5 \%$ and the PPV 98.9\%.

Umbilical artery Doppler measurements were available for 75/76 infected placentas (not performed in a case of intra-uterine fetal demise at $18 \mathrm{wg}$ ) and for all exposed placentas (Supplementary Figure 3). Umbilical artery RI higher than the $95^{\text {th }}$ percentile tended to be more frequent in infected placentas compared to exposed placentas $(12.0 \%$ vs 6.0\%, adjusted for maternal co-morbidities although not significant: $\mathrm{aRR}=1.95$ [95\%CI 0.85-4.19], $\mathrm{p}=0.0945$ ). When considering infected placentas, umbilical artery RI higher than the $95^{\text {th }}$ percentile was more frequent in placentas stemming from fetal loss (3/10, 30.0\%) compared to those stemming from asymptomatic congenital infections (3/49, 6.1\%; $\mathrm{aRR}=4.83$ [95\%CI 1.09-20.64], $\mathrm{p}=0.0292)$, but not more frequent in cases of CZS $(3 / 16$, 18.8\%; $\mathrm{aRR}=3.0$ [95\%CI 0.60-13.09], $\mathrm{p}=0.13)$.

\section{Association between histopathological findings and ZIKV status of the placenta}

Pathological examinations were available for $43 / 76$ (56.6\%) infected placentas and 171/215 (79.5\%) exposed placentas (Figure 1). No differences in baseline maternal characteristics and pregnancy outcomes were observed between these two groups, apart from more frequent fetal loss in congZIKV infections $(\mathrm{p}<0.001)$ (supplementary table). No differences in baseline maternal and pregnancy outcomes were observed whether pathological examination was available or not (data not shown). Among infected placentas (congZIKV), 27/43 (62.8\%; 95\%CI 48.3-77.2) demonstrated pathological anomalies: 5 chorioamnionitis lesions, 9 infarcts, 14 ischemic necrosis with fibrin deposits (INFD), 8 chronic villitis or inter-villitis, 2 subchorionic thrombosis, 2 calcifications. Leukocytic infiltration 
and Hofbauer cell hyperplasia were demonstrated by immunohistochemistry in two placentas of fetuses with CZS (following termination of pregnancy at 27 weeks and stillbirth at 33 weeks). Among exposed placentas (expZKV), 37/215 (21.6\%; 95\%CI 15.5-27.8) demonstrated anomalies at pathological examination: 4 chorioamnionitis lesions, 10 infarcts, 5 INFD, 5 chronic villitis or intervillitis, 5 subchorionic thrombosis and 8 calcifications.

Infected placentas (congZIKV) exhibited a higher risk of any pathological anomalies compared to exposed (expZIKV) placentas $(\mathrm{RR}=2.90$ [95\%CI 2.01-4.19], $\mathrm{p}<0.0001)$, even when adjusting for gestational age at delivery and co-morbidities ( $\mathrm{aRR}=2.60$ [95\%CI 1.40-4.83], p=0.002) (Table 2). A low birthweight/placental weight ratio was also more frequent in infected (congZIKV) placentas compared to exposed (expZIKV) placentas (aRR= 2.78 [95\%CI 1.12-8.78], p=0.035).

When considering infected placentas, pathological anomalies were not observed more frequently in placentas stemming from CZS (8/12, 66.7\%) or fetal loss (8/10, 80.0\%) compared to those stemming from asymptomatic congenital infections (11/21, 52.4\%), after adjusting for gestational age at birth / IUFD and co-morbidities $(\mathrm{aRR}=1.22$ [95\%CI 0.66-2.17] $\mathrm{p}=0.42$ and $\mathrm{aRR}=1.49$ [95\%CI 0.82-2.48] $\mathrm{p}=0.14)$

Among infected placentas (congZIKV), positive RT-PCR at birth were found in 51/58 (87.9; 95\%CI 76.7-95.0) of placentas tested and was not associated with a higher risk of any placental pathology $(p=0.80)$ or low birthweight/placental weight ratio. 


\section{DISCUSSION}

\section{Main results}

In this study, ZIKV-infected placentas were thicker than non-infected placentas after 26 weeks' gestation and placentomegaly was more prevalent and presented earlier in comparison to non-infected placentas, particularly in cases of fetal / neonatal adverse outcomes (CZS and / or fetal loss). Infected placentas also exhibited a higher risk of anatomopathological anomalies than non-infected placentas.

\section{Limitations}

While the accuracy of molecular and serological testing for maternal and congenital ZIKV infection is still under debate, ZIKV status was assessed at multiple time points and on numerous samples ${ }^{15}$, thus reducing the risk of false negative results. Non-infected pregnant women remained negative on ZIKV testing during all trimesters of pregnancy and at delivery ${ }^{14}$. Fetal status of infants born to ZIKV positive and negative pregnant women was assessed on multiple samples, including umbilical cord and neonatal blood, placenta, urine and amniotic fluid and/or cerebro-spinal fluid for symptomatic cases, limiting the risk of misclassification ${ }^{15}$. This potential misclassification would result in a potential underestimation of the observed differences between the three groups. The risk of false positive ZIKV results was limited because pregnant women were tested in the primo-epidemic stage, without significant circulation of other Flaviviridae during this period, and possible cross-reactions were minimized using a micro-neutralizing assay ${ }^{15}$.

\section{Interpretation}

Placentomegaly was observed in $39.5 \%$ of infected placentas and was significantly associated with congenital ZIKV infection. A low birthweight/placental weight ratio was also associated with 
congenital ZIKV infection. Placentomegaly identified via US within a few weeks after infection may be the consequence of placental inflammation due to recent maternal and trans-placental infection ${ }^{10}$. The higher risk of low birthweight/placental weight ratio in the infected group is likely related to the additional impact associated with placentomegaly, which reflects the persistent enlargement of placentas. Placental enlargement could result from fibrinoid deposition and small vascularized villi that form to compensate for in utero hypoxia, recently described in congenital ZIKV infections ${ }^{9}$ and other congenital infections ${ }^{23}$. Placentomegaly in the infected group appeared earlier than in the exposed and control groups, which may be an early sign of trans-placental infection. Placentomegaly was observed in both asymptomatic and symptomatic congenital infections. In fetuses with CZS, placentomegaly appeared after fetal anomalies in one case, at the same time in two cases, and before the appearance of CZS in seven cases (from 2 to 13 weeks before).

The relatively low rate of a low birthweight/placental weight ratio compared to the rate of placentomegaly could also indicate that some of the instances of placentomegaly observed are transient and do not affect placental volume at birth. These transient placentomegalies may be associated with other co-morbidities and natural growth of the placenta, which may be why placentomegaly was identified in the third trimester in the exposed and control groups, and is thus unrelated to ZIKV. Abnormal umbilical Doppler measurements tended to occur more frequently in infected placentas but less so than placentomegaly. These results may indicate that placental infection resulting in placentomegaly does not always lead to placental dysfunction. However, an abnormal umbilical Doppler associated with placentomegaly in infected placentas may predict an acute risk of fetal loss (even if the fetus does not present with growth restriction at diagnosis) ${ }^{24,25}$.

The sensitivity of placentomegaly to predict congenital ZIKV infection is debatable but is similar for other congenital infections ${ }^{23}$. The sensitivity, however is increased when predicting CZS or fetal loss. 
The NPV $>90 \%$ for congenital infection and $>98 \%$ for adverse outcomes of placenta thickness $<40$ mm may help to reassure pregnant patients in poor resource areas where molecular and serological testing are not available during an epidemic peak. Moreover, placental thickness measurement by ultrasound is easy to perform and does not require advanced expertise in comparison to neurosonograms.

It is important to keep in mind that increased placental thickness is not specific to congenital ZIKV infection and is associated with other congenital infections, chromosomal and fetal abnormalities, and maternal pathologies ${ }^{26-29}$.

Compared to exposed placentas, infected placentas exhibited a 2.6-fold increased risk of any histopathological anomaly. Placental inflammation consists of diffuse vascular inflammation, chorioamnionitis lesions, villitis and calcifications, such as described in cCMV infections ${ }^{30}$. ZIKV showed its capacity to specifically infect human placental macrophages and trophoblasts ${ }^{31}$, but placental inflammation was inconsistently described ${ }^{11,32,33}$ and could only occur in early stages of congenital infections ${ }^{34}$. Our study highlighted a higher rate of ischemic necrosis with fibrin deposits (INFD) in ZIKV-infected placentas, which could increase placental growth, such as in Syphilis congenital infections ${ }^{35}$. Only excessive INFD were considered and were found in all cases of fetal loss, however, this finding does not seem to be specific to ZIKV infection as it is also often found in fetal losses of other origins ${ }^{36}$. Subchorionic thrombosis and congestive capillaries observed more often in infected placentas have been previously described in congenital ZIKV infections, and could contribute to fetal hypoxia ${ }^{37}$. We showed Hofbauer cell hyperplasia and leukocytic infiltration in ZIKV-infected placentas, as described by others as a specific finding in ZIKV-infected placentas ${ }^{11,}$ 24, 38 . 
Histopathological examination of the placenta highlights the potential long-term consequences of placental disorders during pregnancy, and the anomalies found may be the result of different pathologies impacting the placenta ${ }^{27}$. We cannot exclude that some of the placental signs observed are partially due to other co-morbidities, however, the similarities in maternal co-morbidities and delivery characteristics between exposed and infected groups reduces the influence of other factors. The main difference between these groups is the rate of fetal loss, but it may be mediated slightly due to the fact that fetal loss is a known consequence of fetal and placental infection by ZIKV ${ }^{5,15}$.

Overall, ZIKV infection of the placenta precedes viral transmission to the fetus. We cannot exclude that ZIKV infection may be restricted to the placenta with no further involvement of the fetus, as described in some CMV infections ${ }^{39}$. Placentomegaly may represent an early sign of congenital infection, before congenital Zika virus syndrome or other adverse outcomes become apparent ${ }^{40}$.

\section{CONCLUSION}

Our study provides a comprehensive description of placental consequences of ZIKV infection. Infected placentas exhibited a higher risk for placentomegaly, low birthweight/placental weight ratio and higher proportion of histopathological anomalies. Although placentomegaly is nonspecific for ZIKV infection, early placentomegaly may represent the first sign of congenital ZIKV infection and should lead to an enhanced prenatal follow-up of ZIKV-exposed pregnancies.

\section{FUNDING}

None 


\section{DISCLOSURES}

All authors have completed the ICMJE uniform disclosure form at www.icmje.org/coi_disclosure.pdf and declare: no support from any organization for the submitted work; no financial relationships with any organizations that might have an interest in the submitted work in the previous three years; no other relationships or activities that could appear to have influenced the submitted work. 


\section{REFERENCES}

1. Rasmussen SA, Jamieson DJ, Honein MA, Petersen LR. Zika Virus and Birth Defects--Reviewing the Evidence for Causality. N Engl J Med 2016; 374: 1981-1987.

2. Oliveira Melo AS, Malinger G, Ximenes R, Szejnfeld PO, Alves Sampaio S, Bispo de Filippis AM. Zika virus intrauterine infection causes fetal brain abnormality and microcephaly: tip of the iceberg? Ultrasound Obstet Gynecol 2016; 47: 6-7.

3. Schaub B, Vouga M, Najioullah F, Gueneret M, Monthieux A, Harte C, Muller F, Jolivet E, Adenet C, Dreux S, Leparc-Goffart I, Cesaire R, Volumenie JL, Baud D. Analysis of blood from Zika virus-infected fetuses: a prospective case series. Lancet Infect Dis 2017; 17: 520-527.

4. Pereira L, Maidji E, McDonagh S, Tabata T. Insights into viral transmission at the uterine-placental interface. Trends Microbiol 2005; 13: 164-174.

5. Panchaud A, Stojanov M, Ammerdorffer A, Vouga M, Baud D. Emerging Role of Zika Virus in Adverse Fetal and Neonatal Outcomes. Clin Microbiol Rev 2016; 29: 659-694.

6. Uenaka M, Morizane M, Tanimura K, Deguchi M, Kanzawa M, Itoh T, Yamada H. Histopathological analysis of placentas with congenital cytomegalovirus infection. Placenta 2019; 75: 62-67.

7. Pereira L, Tabata T, Petitt M, Fang-Hoover J. Congenital cytomegalovirus infection undermines early development and functions of the human placenta. Placenta 2017; 59 Suppl 1: S8-S16.

8. Tsuge M, Hida Al, Minematsu T, Honda N, Oshiro Y, Yokoyama M, Kondo Y. Prospective Cohort Study of Congenital Cytomegalovirus Infection during Pregnancy with Fetal Growth Restriction: Serologic Analysis and Placental Pathology. J Pediatr 2018. DOI:10.1016/j.jpeds.2018.10.003.

9. Hirsch AJ, Roberts VHJ, Grigsby PL, Haese N, Schabel MC, Wang XJ, Lo JO, Liu Z, Kroenke CD, Smith JL, Kelleher M, Broeckel R, Kreklywich CN, Parkins CJ, Denton M, Smith P, DeFilippis V, Messer W, Nelson JA, Hennebold JD, Grafe M, Colgin L, Lewis A, Ducore R, Swanson T, Legasse AW, Axthelm MK, MacAllister $R$, Moses AV, Morgan TK, Frias AE, Streblow DN. Zika virus infection in pregnant rhesus macaques causes placental dysfunction and immunopathology. Nature Communications 2018; 9.

10. Noronha L, Zanluca C, Azevedo ML, Luz KG, Santos CN. Zika virus damages the human placental barrier and presents marked fetal neurotropism. Mem Inst Oswaldo Cruz 2016; 111: 287-293.

11. Rosenberg AZ, Yu W, Hill DA, Reyes CA, Schwartz DA. Placental Pathology of Zika Virus: Viral Infection of the Placenta Induces Villous Stromal Macrophage (Hofbauer Cell) Proliferation and Hyperplasia. Arch Pathol Lab Med 2017; 141: 43-48.

12. Mehrjardi MZ. Is Zika Virus an Emerging TORCH Agent? An Invited Commentary. Virology (Auckl) 2017; 8: 1178122X17708993.

13. Sanz Cortes M, Rivera AM, Yepez M, Guimaraes CV, Diaz Yunes I, Zarutskie A, Davila I, Shetty A, Mahadev A, Serrano SM, Castillo N, Lee W, Valentine G, Belfort M, Parra G, Mohila C, Aagaard K, Parra Saavedra M. Clinical assessment and brain findings in a cohort of mothers, fetuses and infants infected with ZIKA virus. Am J Obstet Gynecol 2018; 218: 440 e441-440 e436.

14. Pomar L, Malinger G, Benoist G, Carles G, Ville Y, Rousset D, Hcini N, Pomar C, Jolivet A, Lambert V. Association between Zika virus and fetopathy: a prospective cohort study in French Guiana. Ultrasound Obstet Gynecol 2017; 49: 729-736.

15. Pomar L, Vouga M, Lambert V, Pomar C, Hcini N, Jolivet A, Benoist G, Rousset D, Matheus S, Malinger G, Panchaud A, Carles G, Baud D. Maternal-fetal transmission and adverse perinatal outcomes in pregnant women infected with Zika virus: prospective cohort study in French Guiana. BMJ 2018; 363: k4431.

16. Picone O, Vauloup-Fellous C, D'Ortenzio E, Huissoud C, Carles G, Benachi A, Faye A, Luton D, Paty MC, Ayoubi JM, Yazdanpanah Y, Mandelbrot L, Matheron S. [Zika virus infection during pregnancy]. J Gynecol Obstet Biol Reprod (Paris) 2016; 45: 415-423. 
17. Vouga M, Musso D, Panchaud A, Baud D. Clinical management of pregnant women exposed to Zika virus. Lancet Infect Dis 2016; 16: 773.

18. Pomar L, Musso D, Malinger G, Vouga M, Panchaud A, Baud D. Zika virus during pregnancy: From maternal exposure to congenital Zika virus syndrome. Prenat Diagn 2019. DOI:10.1002/pd.5446.

19. Grannum PA. Ultrasound Examination of the Placenta. Clin Obstet Gynaecol 1983; 10: 459-473.

20. Dombrowski MP, Wolfe HM, Saleh A, Evans MI, O'Brien J. The sonographically thick placenta: a predictor of increased perinatal morbidity and mortality. Ultrasound Obstet Gynecol 1992; 2: 252-255.

21. Acharya G, Wilsgaard T, Berntsen GK, Maltau JM, Kiserud T. Reference ranges for serial measurements of umbilical artery Doppler indices in the second half of pregnancy. Am J Obstet Gynecol 2005; 192: 937-944.

22. Thompson JM, Irgens LM, Skjaerven R, Rasmussen S. Placenta weight percentile curves for singleton deliveries. BJOG 2007; 114: 715-720.

23. La Torre R, Nigro G, Mazzocco M, Best AM, Adler SP. Placental enlargement in women with primary maternal cytomegalovirus infection is associated with fetal and neonatal disease. Clin Infect Dis 2006; 43: 994-1000.

24. Simoni MK, Jurado KA, Abrahams VM, Fikrig E, Guller S. Zika virus infection of Hofbauer cells. Am J Reprod Immunol 2017; 77.

25. Chen KH, Chen LR, Lee YH. The Role of Preterm Placental Calcification in High-Risk Pregnancy as a Predictor of Poor Uteroplacental Blood Flow and Adverse Pregnancy Outcome. Ultrasound Med Biol 2012; 38: 1011-1018.

26. Elchalal U, Ezra Y, Levi Y, Bar-Oz B, Yanai N, Intrator O, Nadjari M. Sonographically thick placenta: a marker for increased perinatal risk--a prospective cross-sectional study. Placenta 2000; 21: 268-272.

27. Benirschke K KP. Pathology of the human placenta. (4th ed. edn). Srpinger: New York, 2000. 28. Ghosh A, Tang MH, Lam YH, Fung E, Chan V. Ultrasound measurement of placental thickness to detect pregnancies affected by homozygous alpha-thalassaemia-1. Lancet 1994; 344: 988-989.

29. Harper MA, Ruiz C, Pettenati MJ, Rao PN. Triploid partial molar pregnancy detected through maternal serum alpha-fetoprotein and HCG screening. Obstet Gynecol 1994; 83: 844-846.

30. Garcia AG, Fonseca EF, Marques RL, Lobato YY. Placental morphology in cytomegalovirus infection. Placenta 1989; 10: 1-18.

31. Quicke KM, Bowen JR, Johnson EL, McDonald CE, Ma H, O'Neal JT, Rajakumar A, Wrammert J, Rimawi BH, Pulendran B, Schinazi RF, Chakraborty R, Suthar MS. Zika Virus Infects Human Placental Macrophages. Cell Host Microbe 2016; 20: 83-90.

32. van der Eijk AA, van Genderen PJ, Verdijk RM, Reusken CB, Mogling R, van Kampen JJ, Widagdo W, Aron GI, GeurtsvanKessel CH, Pas SD, Raj VS, Haagmans BL, Koopmans MP. Miscarriage Associated with Zika Virus Infection. N Engl J Med 2016; 375: 1002-1004.

33. Esquivel M, Avaad-Portari E, Vasconcelos ZC, Moreira ME, Gaw SL. Vertical transmission and placental pathology of twin pregnancies from Zika virus infected mothers. Am J Obstet Gynecol 2018; 218: $\mathrm{S}_{520} \mathrm{~S}_{520 .}$

34. Schwartz DA. Viral infection, proliferation, and hyperplasia of Hofbauer cells and absence of inflammation characterize the placental pathology of fetuses with congenital Zika virus infection. Arch Gynecol Obstet 2017; 295: 1361-1368.

35. Kittipornpechdee N, Hanamornroongruang S, Lekmak D, Treetipsatit J. Fetal and Placental Pathology in Congenital Syphilis: A Comprehensive Study in Perinatal Autopsy. Fetal Pediatr Pathol 2018. DOI:10.1080/15513815.2018.1485798. 1-12.

36. Ritter JM, Martines RB, Zaki SR. Zika Virus: Pathology From the Pandemic. Arch Pathol Lab Med 2017; 141: 49-59. 
37. Chimelli L, Melo ASO, Avvad-Portari E, Wiley CA, Camacho AHS, Lopes VS, Machado HN, Andrade CV, Dock DCA, Moreira ME, Tovar-Moll F, Oliveira-Szejnfeld PS, Carvalho ACG, Ugarte ON, Batista AGM, Amorim MMR, Melo FO, Ferreira TA, Marinho JRL, Azevedo GS, Leal J, da Costa RFM, Rehen $\mathrm{S}$, Arruda MB, Brindeiro RM, Delvechio R, Aguiar RS, Tanuri A. The spectrum of neuropathological changes associated with congenital Zika virus infection. Acta Neuropathol 2017; 133: 983-999.

38. Maykin M, Avaad-Portari E, Esquivel M, Pereira JP, Fisher SJ, Moreira ME, Nielsen-Saines K, Brasil P, Gaw SL. Placental Histopathologic Findings in Zika-infected Pregnancies. Am J Obstet Gynecol 2018; 218: S520-S521.

39. Trincado DE, Munro SC, Camaris C, Rawlinson WD. Highly sensitive detection and localization of maternally acquired human cytomegalovirus in placental tissue by in situ polymerase chain reaction. $J$ Infect Dis 2005; 192: 650-657.

40. Miwa I, Sase M, Torii M, Sanai H, Nakamura Y, Ueda K. A thick placenta: a predictor of adverse pregnancy outcomes. Springerplus 2014; 3: 353.

\section{Figure legends}

Figure 1: Flow-chart

Figure 2: Prenatal thickness of congZIKV, expZIKV and control placentas, by gestational age

Figure 3: Apparition of placentomegaly, according to gestational age

Figure 4: Apparition of placentomegaly across infected placentas, according to fetal / neonatal outcome

Supplementary Figure 1: Non parametric Lowess curves of the kinetic of placental thickness by ZIKV status

Supplementary Figure 2: Placentomegaly related to congenital Zika infection, at 22 weeks' gestation

Supplementary Figure 3: Fetal growth, umbilical artery RI and kinetics of the placental thickness, according to gestational age and ZIKV-status of the placenta

Fetal growth estimation was calculated using Hadlock $3(\log 10 \mathrm{EPF}=1.326-0.00326(\mathrm{AC})(\mathrm{FL})+$ $0.0107(\mathrm{HC})+0.0438(\mathrm{AC})+0.158(\mathrm{FL})$, the 3rd percentile curve refers to Intergrowth 21 st charts.

Placentas associated with Small for gestational age (SGA) fetuses (confirmed on birthweight) were not more frequent in case of ZIKV-infection (1/59, 1.7 \%) compared to ZIKV-exposed (5/196, $2.5 \%)$ or

controls $(7 / 334,2.1 \%)$. When considering thick placentas $(>40 \mathrm{~mm})$, SGA fetuses were not more frequent in infected placentas $(1 / 29,3.4 \%)$ compared to exposed placentas $(2 / 37,5.4 \%)$ or controls $(2 / 24,8.3 \%)$ 


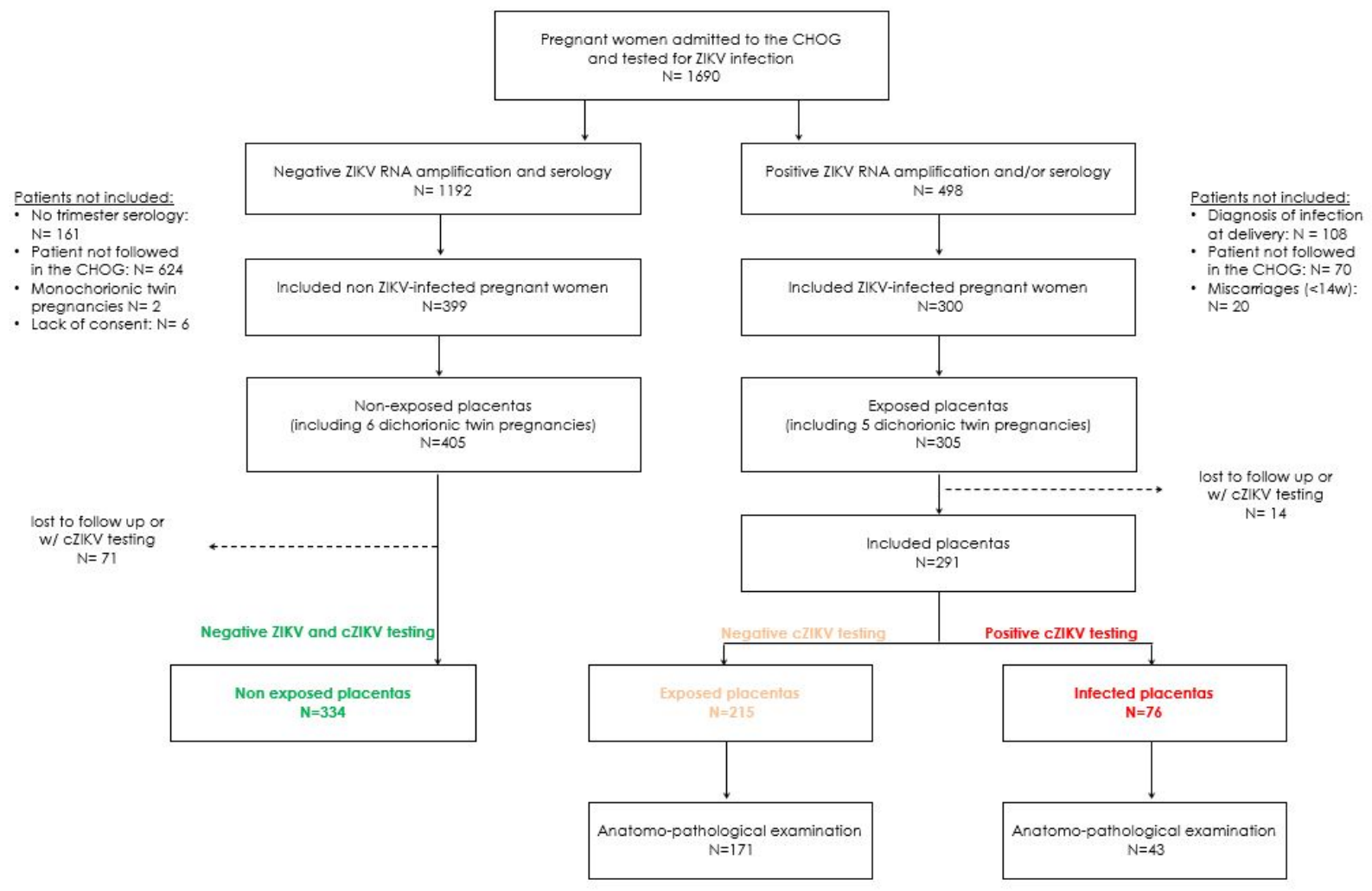

Figure 1: Flow-chart 


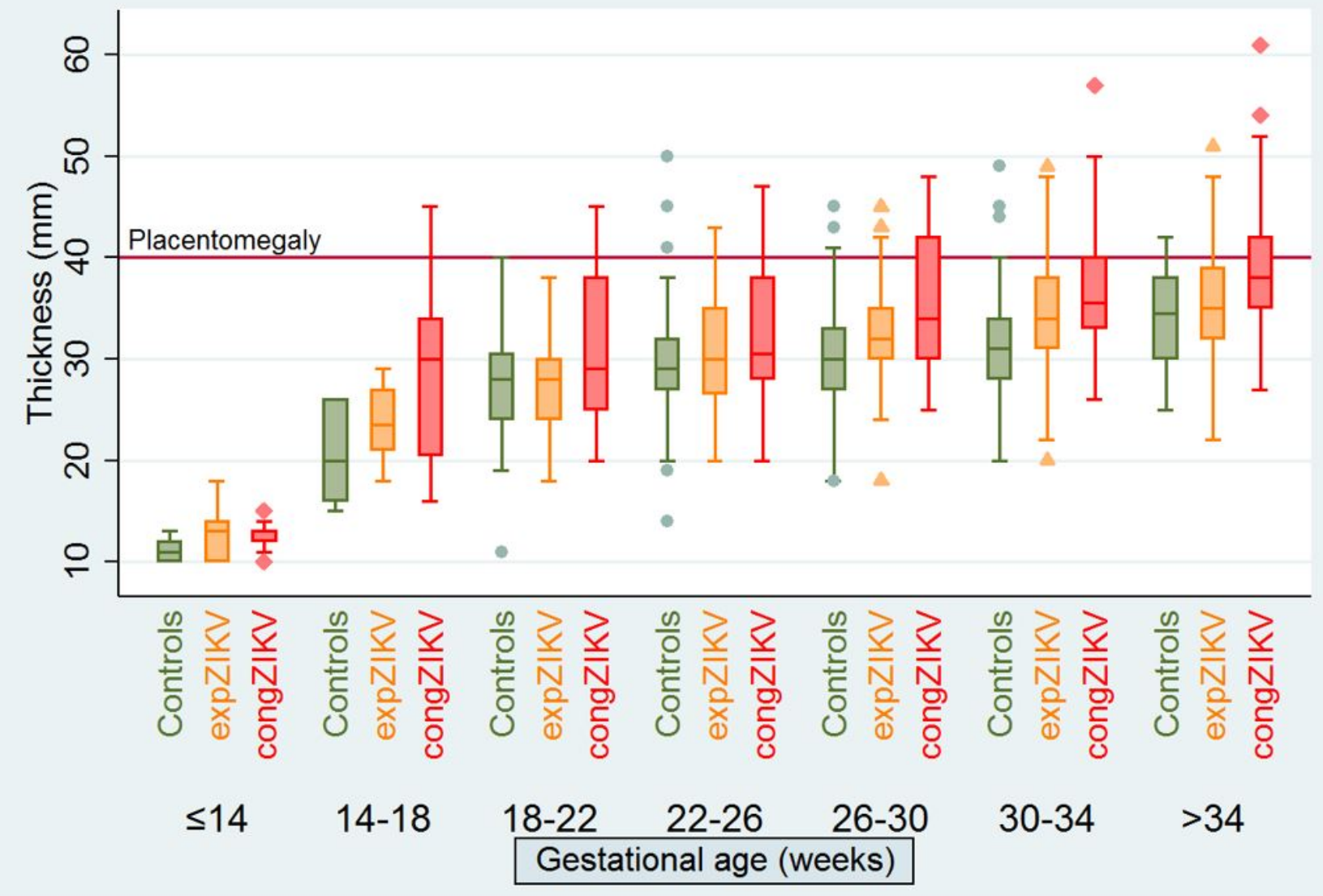

Figure 2: Prenatal thickness of congZIKV, expZIKV and control placentas, by gestational age 


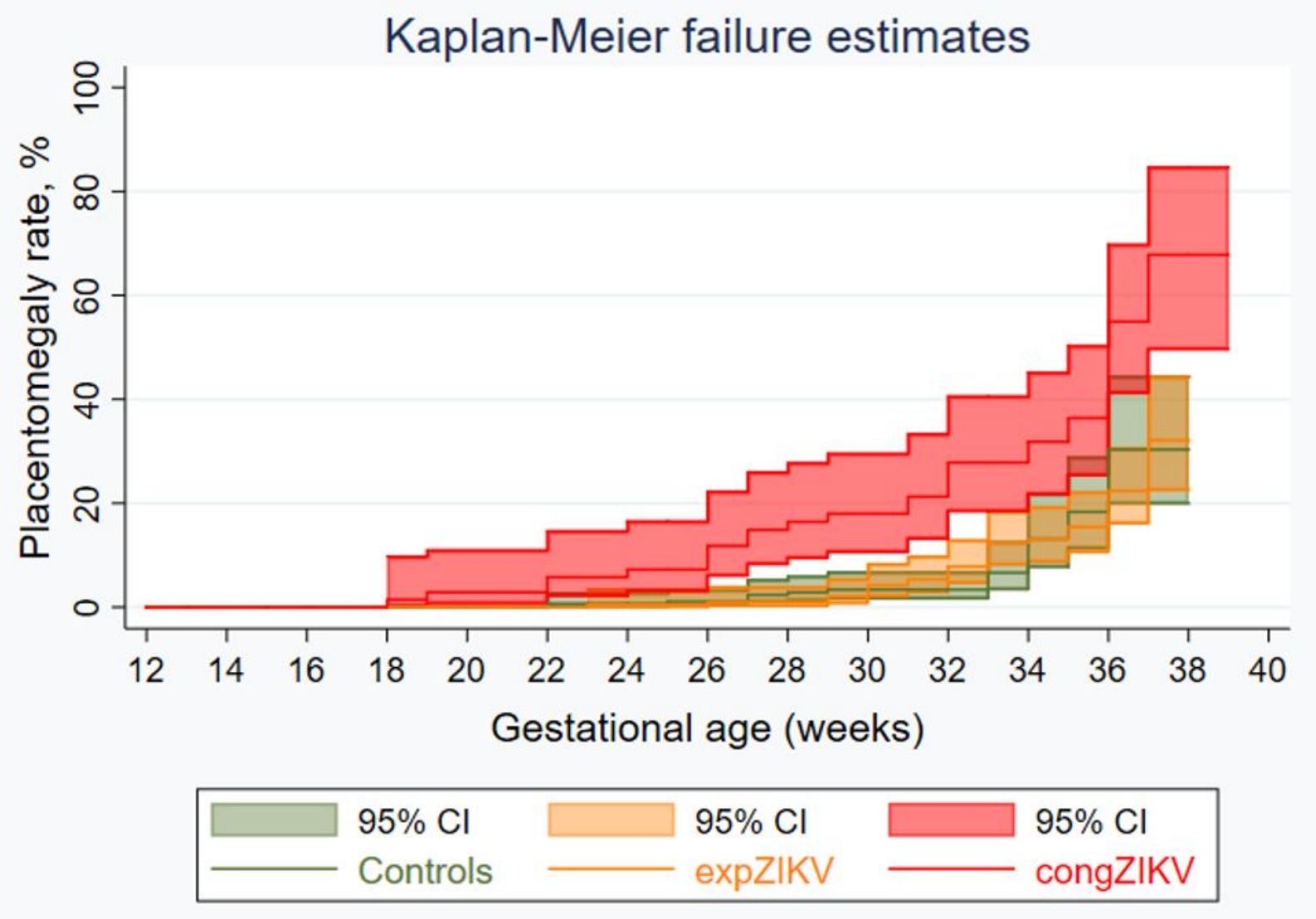

Figure 3: Apparition of placentomegaly, according to gestational age 


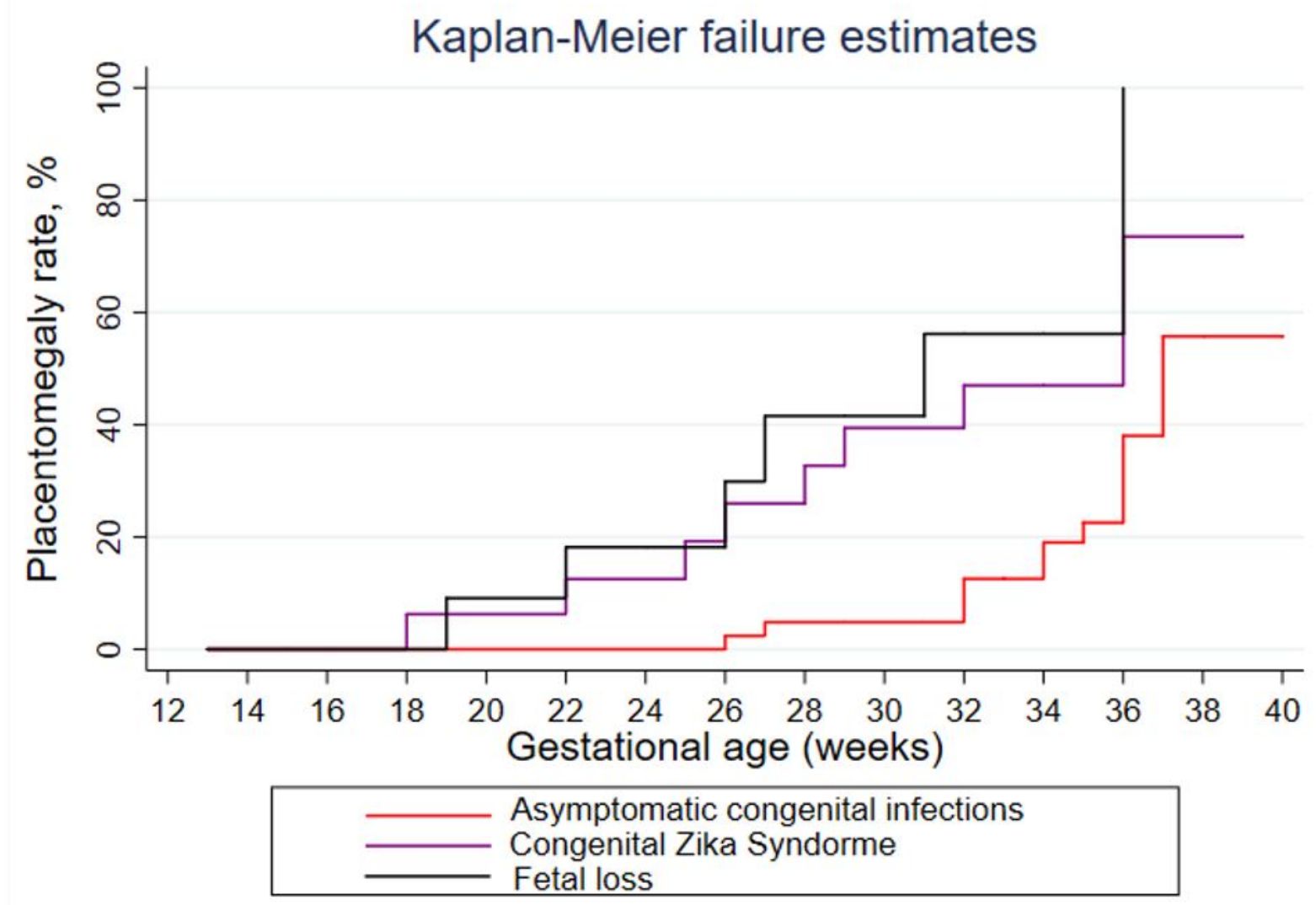

Figure 4: Apparition of placentomegaly across infected placentas, according to fetal / neonatal outcome 
Table 1: Clinical Characteristics

\section{Maternal age}

Median - y.o. (IQR)

Age $>35$ y.o.

Any maternal comorbidities ${ }^{\pi}$ - no (\%)

Diabetes (previous or gestational)

Vascular pathologies

Thrombophilia

Anemia

Co-infections*

Lead poisoning

Alcohol consumption

Others**

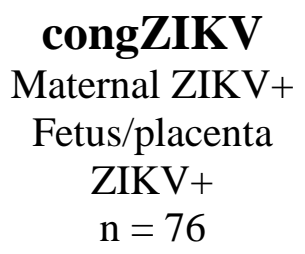

12 (15.8)

21 (27.6)

2 (2.6)

$6 \quad(7.9)$

2 (2.6)

4 (5.3)

3 (3.9)

2 (2.6)

1 (1.3)

3 (3.9)

1 (1.3)

$2(2.6)$

117 (54.4)

47 (61.8)

27 (35.5)

\section{Controls}

Maternal ZIKV+ Maternal ZIKV-

Fetus/placenta ZIKV-

$\mathrm{n}=215$
Fetus/placenta

ZIKV-

$\mathrm{n}=334$

\section{Dichorionic twins, no (\%)}

\section{Risk of fetal aneuploidy}

$$
\begin{aligned}
& >1 / 250-\text { no/total no (\%) } \\
& <1 / 250 \\
& \text { Late follow-up (> 14wg) }
\end{aligned}
$$

\section{Trimester of maternal infection}

diagnosis

$\mathrm{T} 1$
$\mathrm{~T} 2$
$\mathrm{~T} 3$

$\begin{array}{llrl}16 & (21.0) & 52 & (24.2) \\ 44 & (57.9) & 111 & (51.6) \\ 16 & (21.0) & 52 & (24.2)\end{array}$

Number of prenatal US, median (minmax)

3 (1.4)

42 (19.5)

79 (23.7)

$17 \quad(5.1)$

19 (5.7)

3 (0.9)

11 (3.3)

9 (2.7)

8 (2.4)

$7 \quad(2.1)$

$8 \quad(2.4)$

6 (1.8)

$\begin{aligned} 14 & (4.2) \\ 26 & (79.0) \\ 4 & \\ 56 & (16.8)\end{aligned}$

T1

T3
$4 \quad(1-7)$
$4 \quad(1-7)$
$3 \quad(1-6)$ 


\section{Fetal loss}

no $(\%)$

11 (14.5)

$1(0.5)$

4 (1.2)

Gestational age - Median - WG (IQR)

$25 \quad(18-32) \quad 33 \quad /$

$33 \quad(28-35)$

\section{Delivery}

Gestational age - Median - WG (IQR)

$38 \quad(35-39) \quad 38 \quad(37-39) \quad 37 \quad(35-39)$

GA $<37$ WG - no (\%)

$12 / 62 \quad(19.4)$

Birthweight $<$ P3 - no (\%)

$1 / 59 \quad(1.7)$

$\begin{array}{rlr}24 / 2 & & 35 \\ 14 & (11.2) & / 3 \\ & & 34 \\ 5 & & 7 / \\ 1196 & (2.5) & 33 \\ & & 4\end{array}$

\section{Fetal / neonatal findings suggestive of}

CZS, no (\%)

$16 / 76 \quad(21.1)$

4

${ }^{\pi}$ Including patients with multiple comorbidities

* congZIKV: two active hepatitis B infections. expZIKV: two HIV, two primary toxoplasmosis, one HTLV, one Coxsackie virus, two

primary VZV and one leptospirosis . Controls: two primary toxoplasmosis, one primary $\mathrm{CMV}$, three HIV and three primary VZV

** Anti-Lea alloimmunisation, malnutrition, vitamin K deficit, increased HCG levels, history of mucopolysaccarosis

"congZIKV": congenital ZIKV-infections

"expZIKV": maternal ZIKV-infection without congenital infection

"Controls": negative maternal, placental and neonatal ZIKV-testing

CZS: congenital Zika syndrome

IQR: interquartile range

WG: weeks' gestation 
Table 2: Placental Outcomes

Placentomegaly $>40 \mathrm{~mm}$, no $(\%$, 95IC)

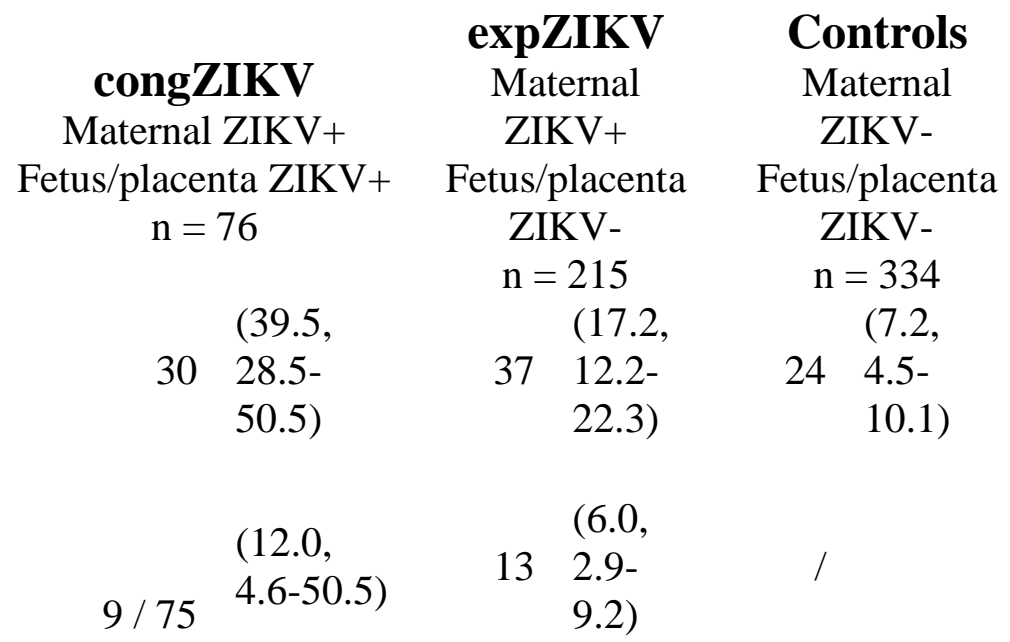

\section{Birthweight / placental weight} ratio

Umbilical artery RI > p95, no $(\%, 95 I C)$

9 / 75 4.6-50.5)

(5.9,

(16.3, $\quad 10 / \quad 2.3-$

$7 / 43 \quad 5.3-27.3) \quad 171 \quad 9.4)$

(8.2,

$(9.3,0.6-\quad 14 / \quad 4.1-$

$\begin{array}{llll}4 / 43 & 18.0) & 171 & 12.3)\end{array}$

> p97, n (\%, 95IC)

Pathology findings - no/total (\%, 95IC)

Chorioamnionitis lesions

Infarcts

Ischemic necrosis with fibrin deposits

Villitis

Chorionic villitis with congestive capillaries

Subchorionic trombosis
(62.8, 48.327 / $43 \quad 77.2$ )

(21.6, 37 / 15.5-

$17127.8)$

(2.3,

(11.6, $\quad 0.1-$

$5 \quad 2.1-21.2) \quad 4 \quad 4.6)$

(5.9,

(20.9, 2.3-

$9 \begin{array}{llll}9.8-33.1) & 10 & 9.4)\end{array}$

(32.6, (2.9,

18.6- $0.4-$

14 46.6) $\quad 5 \quad 5.4)$

(2.9,

$0.4-$

(18.6,

$8 \begin{array}{llll}8 & 7.0-30.2) & 5 & 5.4)\end{array}$

(2.3,

(13.9,

$0.1-$

$\begin{array}{llll}6 & 3.6-24.3) & 4 & 4.6)\end{array}$

(2.9,

(4.6, 1.6- $\quad 0.4-$

2 10.9)

$5 \quad 5.4)$ 
Calcifications

Leukocytic infiltration

Hofbauer cells hyperplasia

Positive RT-PCR - no/total (\%, 95IC)

With placentomegaly

With umbilical RI > p95

With pathology findings
(4.7,

(11.6, $\quad 1.5-$

$5 \quad 2.1-21.2) \quad 8 \quad 7.9)$

(4.6, 1.6-

2 10.9) 0

(4.6, 1.6-

2 10.9)

0

(87.9, 76.7-

$51 / 58$ 95.0)

$0 / 174$

$0 / 33$

4

(51.0,

37.3-

$26 / 51 \quad 64.7)$

(11.8,

$6 / 51 \quad 2.9-20.6)$

(60.0,

43.8-

$21 / 35 \quad 76.2)$

"congZIKV": congenital ZIKV-infections

"expZIKV": maternal ZIKV-infection without

congenital infection

"Controls": negative maternal, placental and neonatal ZIKV-testing

RI: resistance index 